\title{
HYDROTHERMAL OPTIMAL POWER FLOW BASED ON A COMBINED LINEAR AND NONLINEAR PROGRAMMING METHODOLOGY
}

\author{
H. Habibollahzadeh * G.X. Luo A. Semlyen \\ Department of Electrical Engineering \\ University of Toronto \\ Toronto, Ontario, Canada \\ * At present with Ontario Hydro
}

Keywords: Optimal Power Flow, Hydraulic System, Thermal System, Method of Feasible Directions.

Abstract - The paper considers a new formulation of Optimal Power Flow (OPF) that makes it eminently suitable for accurate incremental modeling. Zoutendijk's method of feasible directions for solving the nonlinear programming problems is adapted for the solution of the OPF. Hydraulic modeling of systems with considerable share of hydraulic generation is also considered. The method is very efficient as it is designed to exploit the special structure of the problem.

\section{INTRODUCTION}

The computation of active and reactive Optimal Power Flow (OPF) has received widespread attention in the last decade [1]-[16]. It is of current interest to many utilities and has been identified as one of the most important operational needs. Real-time application in particular requires the computer codes to be fast and robust, high accuracy having less importance. In this type of problems the choice of method lies between two main approaches: nonlinear and linear programming techniques.

Nonlinear programming techniques [1]-[7],[11]-[16],[26] have been used for accurate active and reactive OPF models. These methods had several drawbacks in the earlier applications, including slow convergence, complexity, and difficulties involved in handling constraints and in adapting to different problems. These problems have been addressed and solved to a great extent in the more recent publications.

The LP method has been reported to be reliable and fast in solving linearized OPF models [8]-[10],[12]. It is very convenient for handling the constraints. But on the other hand, it produces solutions that are at the comers of the linearized feasible region while the nonlinear objective could lie anywhere within the feasible region. An important drawback is that LP allows only for a lincar objective function. Oscillatory bchavior may also occur if the LP is iterated without good linearization of the constraints.

The work reported in this paper focuses on the active problem even though it can be applied equally to the reactive power problem. It is based on the combination of the two methods, to take advantage of the strong points in one to compensate for the shortcomings of the other. One previous paper [15] was based on such an idea and it used the LP technique to help the Generalized Reduced Gradient technique in a feasibility adjustment step. In the work presented here, Zoutendijk's feasible directions method [21] for solving nonlinear programming problems has been used. In this method an incremental model using the gradient at an existing solution is employed. A small linear domain around this solution is chosen to ensure good linearization. The original constraints that bind in this small region are included in the incremental model. This model is

38 SM 729-6 A paper recommended and approved by the IEEE Power System Engineering Committee of the IEEE Power Engineering Society for presentation at the IEEE/PES 1988 Summer Meeting, Portland, oregon, July $24-29,1988$. Manuscript submitted February 1, 1988; made available for printing June 6,1988 . a sparse LP with embedded network structure. Solution of the LP produces a feasible improving direction. The nonlinear objective is then optimized along this feasible direction within the original nonlinear constraints. The process is continued at the new solutions obtained until the problem converges.

Several modifications have been made to avoid the general problems in existing optimization techniques, mentioned earlier. First, to exploit the speed of the LP technique, the first solution is produced using a linear model in which a piecewise linear approximation of the cost curve and linearization of the nonlinear constraints is employed. This will result in a near optimum solution that can be used to start the nonlinear programming technique. Second, a branch oriented formulation of OPF is used as opposed to a nodal one to provide an accurate linearization of the problem. At least one paper [14] has addressed this problem and has given a good linearization model. Third, the sparsity and the embedded network structure of the constraints are exploited to speed up the solution technique. Fourth, the method of parallel tangents is used to speed up the convergence of the nonlinear technique. The procedure developed in this work is capable of starting from an infeasible initial solution.

The hydraulic system is not normally included at the instantaneous OPF level. It involves time due to the water energy storage and is normally considered in daily, weekly or seasonal optimizations [22],[23]. One previous work [25], in which an approximate hydraulic model is considered, combines the daily optimization and OPF. Hydraulic modeling in OPF for systems with high share of hydraulic generation is essential. The present work includes the hydraulic system in the OPF.

Test results from the application of the proposed technique are presented to demonstrate the capability of the solution technique.

\section{PROBLEM FORMULATION}

The Optimal Power Flow (OPF), as formulated in this work, has the following structure:

Minimize

$$
f(x)
$$

Subject to

$$
\begin{gathered}
A_{1} x=b_{1} \\
h(x)=b_{2} \\
g(x) \leq b_{3} \\
x_{\min } \leq x \leq x_{\max }
\end{gathered}
$$

The variable vector $x$ consists of real and imaginary parts of voltages and currents on all network branches, longitudinal and transversal, plus the active generated powers (these active powers are further divided into components in the case of piecewise linear approximation of the thermal production costs).

The branch oriented formulation of OPF when compared with the conventional nodal formulation has several important advantages, even though the size of the problem grows due to the introduction of more 
variables. First, the representation of the system equations is simplified and the model becomes very sparse and has an embedded network structure. Second, the technique becomes very efficient for constrained OPF problems where new constraints bind during the optimization. These constraints can be added in a simple manner with minimal effort and computational time requirement. Third, the special structure of this formulation makes it possible to apply special fast algorithms to solve the problem. These points are considered in the following sections.

\section{The Objective Function}

The objective in the active Optimal Power Flow Problem is to minimize the production cost of the thermal system. The production cost curve for a thermal plant is nonlinear and is normally approximated as a quadratic curve. In this work, the objective is considered differently in two stages. In the first stage a piecewise linear approximation of the thermal cost curves is used. This approximation connects the minimum and maximum production levels through the most efficient operating points corresponding to different number of generators in operation, as shown in figure 1.

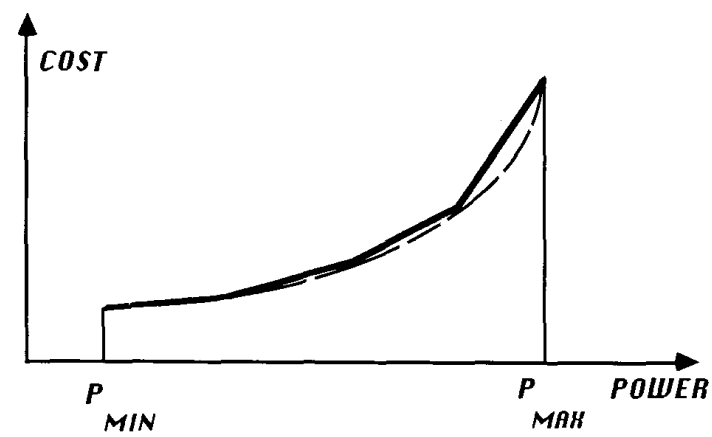

Figure 1. Piecewise linear approximation of cost curve for a typical thermal unit.

In this stage, the active power generated from a thermal plant must be divided into components, each corresponding to a line segment, as follows

$$
P_{i}=\sum_{j} P_{i j}+P_{\min }
$$

where $P_{i}$ is the power production from the thermal plant $i$ and $P_{i j}$ represents its components corresponding to the different line segments in the piecewise linear approximation (the summation is over the number of line segments).

The quadratic approximation, as shown in figure 1 by the dotted curve, is considered in the second stage.

\section{The Linear and Nonlinear Constraints}

The linear equality constraints (2) consist of Kirchhoff's voltage and current laws and Ohm's law. The coefficient matrix corresponding to these constraints is very sparse and has an embedded network structure. These constraints constitute the major part of the total constraints involved in the problem. This is very important for the method considered in this paper.

The nonlinear equality constraints (3) represent the active and reactive power definitions in terms of voltages and currents. This set constitutes a very small part of the constraints.

The nonlinear inequality constraints (4) reflect the usual operational limits. The number of constraints involved in this set is rather large. But the number of binding constraints is usually very low. These are the only ones required to be eventually considered in the optimization.

\section{A 5-Bus Example}

In this section, the simple 5-bus system shown in figure 2 is considered in order to present some further details of the problem formulation.

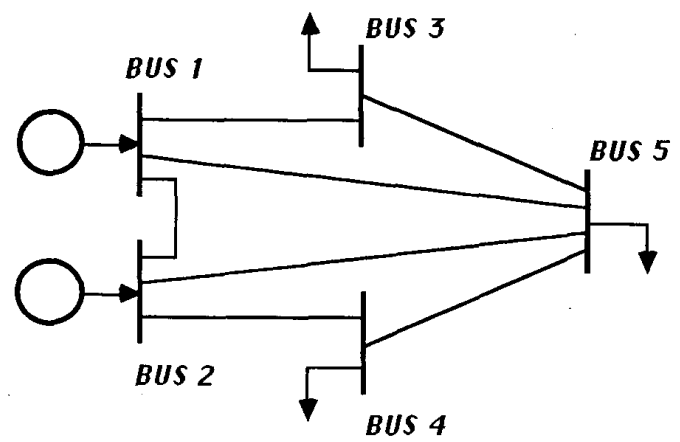

Figure 2. A simple 5-bus system

The linear constraints for this system consist of three different sets of equations. The first set contains Kirchhoff's current law at the five buses for the real and imaginary parts of the complex currents (two equations per bus):

$$
\Sigma I_{i}=0, \quad \Sigma{ }^{\prime \prime}{ }_{i}=0
$$

(the summation is over all currents incident to a node. "and " represent the real and imaginary parts of a complex variable, respectively).

The second set consists of Kirchhoff's voltage law in the seven loops of the system (some involving the ground) for the real and imaginary parts of the voltages (one equation per line):

$$
\Sigma V^{\prime}{ }_{i}=0, \quad \Sigma V^{\prime \prime}{ }_{i}=0
$$

(the summation is over all the voltages in one loop).

The third set consists of Ohm law for the components of the network:

$$
V=Z I
$$

where $V$ and $I$ are the vectors containing the complex voltages across and the complex currents through the network components. $Z$ is a diagonal matrix containing the impedance of the components. This set will contain two equations per line and two equations per bus (for the shunt admittances).

The nonlinear equality constraints consist of two sets of equations. The first set contains the nonlinear power equations at the load buses in terms of the complex voltages and currents (two equations per load bus):

$$
\begin{aligned}
& P_{i}=\operatorname{Re}\left(V_{i} I^{*}\right)=V^{\prime}{ }_{i} I_{i}{ }+V^{\prime}{ }_{i} I^{\prime \prime}{ }_{i} \\
& Q_{i}=\operatorname{Im}\left(V_{i} I^{*}\right)=V^{\prime \prime}{ }_{i} I_{i}{ }_{i}-V^{\prime}{ }_{i} I^{\prime}{ }_{i}
\end{aligned}
$$

The second set consists of equations representing the real power production at the generator buses with respect to the complex voltages and currents (one equation per generator bus)

$$
P_{i}=\operatorname{Re}\left(V_{i} I^{*}{ }_{i}\right)=V^{\prime}{ }_{i} I^{\prime}{ }_{i}+V^{\prime \prime}{ }_{i} I^{\prime \prime}{ }_{i}
$$

For the case of piecewise linear approximation, the power is replaced by its components:

$$
P_{\min }+\sum_{j} P_{i j}=\operatorname{Re}\left(V_{i} I_{i}\right)
$$

The nonlinear inequality constraints reflect the usual operational limits. For the simple 5-bus system, this set contains the lower and upper limits for the bus voltages, for the active and reactive power generations and the transmission line limits (two equations per load bus, four equations per generation bus and one equation per line):

$$
\begin{aligned}
& V_{i \text { min }} \leq V_{i} \leq V_{i \max } \\
& P_{i \text { min }} \leq P_{i} \leq P_{i_{\max }}
\end{aligned}
$$




$$
\begin{gathered}
Q_{i \min } \leq Q_{i} \leq Q_{i \max } \\
I_{l} \leq I_{l_{\max }}
\end{gathered}
$$

Tap changing and phase shifting transformers are modeled in the conventional way.

\section{HYDRAULIC SYSTEM}

The optimal operation planning of the electric power system is normally divided into several subproblems which are computationally manageable and each subproblem provides the answer to a different aspect of the total problem. The different subproblems are:

1) Long-term production planning (2-3 years of optimization horizon, monthly discretization intervals)

2) Seasonal production planning (yearly horizon, weekly intervals)

3) Weekly production planning (weekly horizon, 4-6 hours intervals)

4) Daily production planning (daily horizon, hourly intervals)

5) Optimal power flow (instantaneous formulation)

The hydraulic system involves time because of the water energy storage. The main economical gains from this system are at the daily or higher optimal scheduling levels [22],[23]. Therefore, the hydraulic production has been always treated as constant in the OPF. In fact this is not valid and the hydraulic system can have a considerable effect in a constrained OPF. For example, a small change in a nearby hydraulic production can relax a binding transmission limit instead of a remote expensive thermal unit required for the same purpose.

The solution of each production planning subproblem provides some of the input required at the next lower level. For example, the daily production planning provides the thermal unit commitment and the hydraulic generations for the OPF. It is shown in this section that other hydraulic information, such as hydraulic unit commitment and water values from the daily scheduling, can be used to allow variable hydraulic generation in OPF.

In this work, a daily hydraulic scheduling program based on network flow algorithms [23] is used to define the hourly hydraulic productions, dual values (water values) and the possible variation limits. The three plant system in figure 3 is used to demonstrate the technique.

The first plant has 3 generators and is connected to bus 1 , the second one has 2 generators and it is connected to bus 2 . The third plant has 3 generators and it is connected to bus 3 .

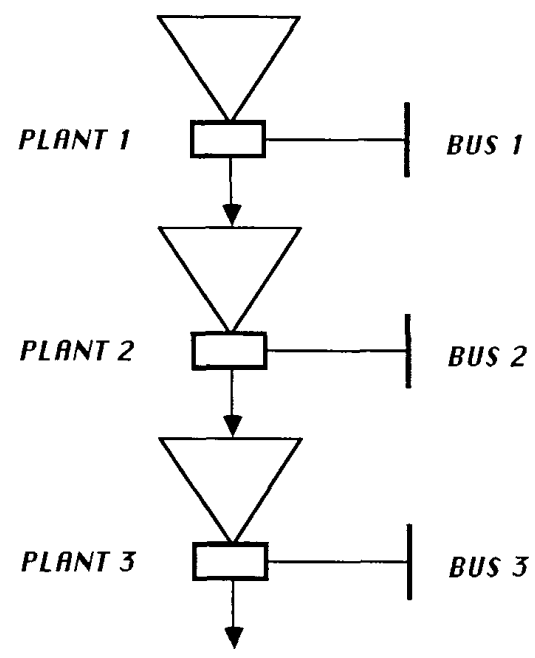

Figure 3. Three plants hydraulic system.

A network formulation of this problem for a daily scheduling is shown in figure 4 . The horizontal arcs indicate the water saved in the reservoirs from one hour to the next. The vertical arcs are the discharges through different turbines and the efficiencies of the corresponding gen- crators are reflected on them. The dual variables corresponding to the flow balance at different nodes in figure 4 are the water values for the reservoirs during the corresponding hours and reflect the sensitivity of hydraulic benefits to flow changes (and the corresponding production changes).

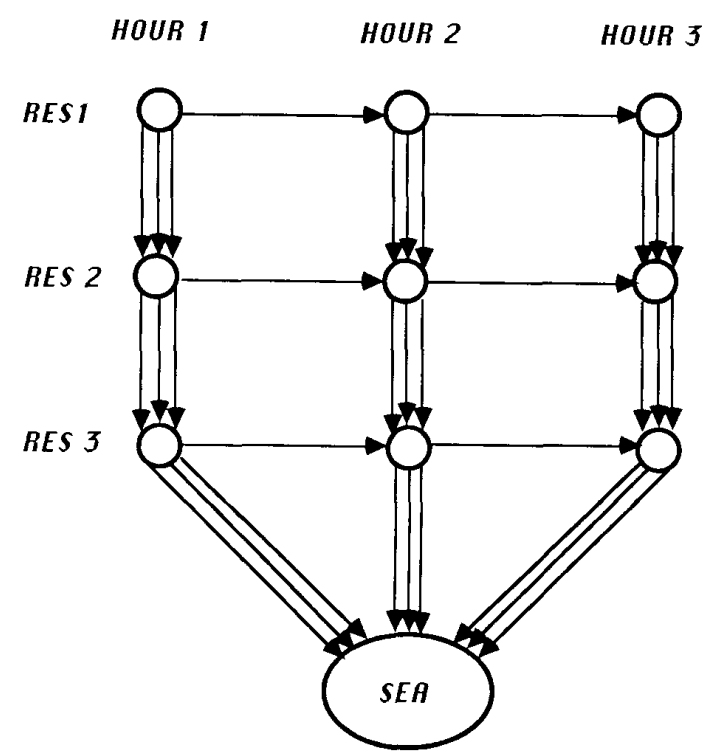

Figure 4 The network representation of the hydraulic system.

The hydraulic unit commitments obtained at the daily level are assumed fixed in OPF. A marginal production variation from the values obtained in the daily scheduling is allowed for each plant located at a node of figure 4 . The variation limit was chosen to be equal to one third of the last unit committed in each plant. This is small enough to keep the dual values valid.

An increase of flow in a vertical arc in figure 4 (a generation unit discharge), such as the third arc from reservoir 1 to 2 during hour 1 , is considered for the sake of explanation. This increase will result in moving a certain amount $\alpha$ of water from rescrvoir 1 during hour 1 , with water value $\pi_{1}$, to reservoir 2 during hour 1 , with water value $\pi_{2}$. The value of the water moved from an upstream reservoir to a lower one is decreased by:

$$
\text { loss }=\alpha\left(\pi_{1}-\pi_{2}\right)
$$

The corresponding gain depends on the efficiency $\eta$ of the generation unit to produce power from the displaced water and the benefits $\lambda$ obtained from the power production:

$$
\text { gain }=\lambda \eta \alpha
$$

If this gain dominates over the corresponding loss, then such a hydraulic production change is beneficial in OPF. Therefore, hydraulic productions are modeled with the corresponding costs and become similar to the thermal units in the OPF solution.

It should be noted that this hydraulic model can be also included in other conventional OPFs.

\section{OPTIMIZATION}

The optimization consists of four steps as shown in figure 5 . In the first step, an initial solution is obtained by fixing the control variables at the generation buses to some predefined values and employing a standard power flow technique. The next three steps are performed from this initial solution using an incremental model. These steps are discussed in the next subsections. 


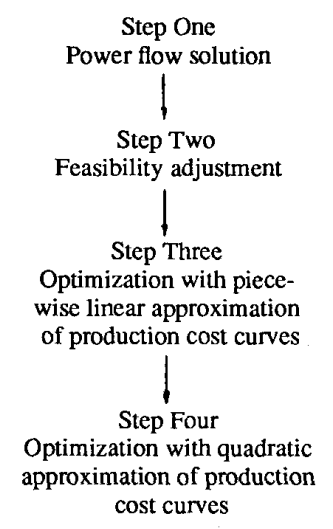

Figure 5. Optimization flow chart

The Incremental Model

The nonlinear equations can be linearized around any existing solution using Taylor's series:

$$
\left.f(x)=f\left(x^{o}\right)+\sum_{i} \frac{\partial f}{\partial x_{i}}\right]_{o} \Delta x_{i}
$$

The linearization of equations (1)-(5) around an existing solution results in:

Minimize

$$
\nabla f\left(x^{\circ}\right) \Delta x
$$

Subject to

$$
\begin{gathered}
A_{1} \Delta x=b_{1}^{\prime} \\
\nabla h\left(x^{o}\right) \Delta x=b_{2}^{\prime} \\
\nabla g\left(x^{o}\right) \Delta x \leq b_{3}^{\prime} \\
\Delta x \varepsilon \Omega
\end{gathered}
$$

where $\Omega$ is a closed domain around the existing solution to ensure that the linearization errors are small. Solution of this incremental problem will produce a feasible improving direction.

The $\Omega$ domain plays an important role in the efficiency and convergence of the optimization technique considered in this work. This domain can not be limited with the conventional nodal approach and this is the reason for the lack of any previous work in the literature in the application of the feasible direction optimization techniques to OPF.

The branch oriented formulation presented above can limit the $\Omega$ domain properly and results in excellent feasible optimum directions. A test was performed on the effect of the reduction of variables, where part of the variables were eliminated by simple algebraic operations. It was noticed that the quality of the directions produced deteriorated from the feasibility and optimality points of view.

This problem can be demonstrated by the simple example shown in figure 6 . The power balance at bus 1 is;

$$
p_{1}=p_{12}+p_{13}
$$

Using the conventional nodal approach our variables are $p_{i}$ 's and $v_{i}$ 's. Therefore, the incremental variables to be limited are $\Delta p_{i}$ 's and $\Delta v_{i}$ 's. Unfortunately, this can only limit variable $p_{1}$ properly but not $p_{12}$ and $p_{13}$. Since the later variables can vary more than desired, the linearization will introduce greater error than anticipated.

In the expanded branch oriented form we have:

$$
\operatorname{Re}\left(V_{1} I_{1}^{*}\right)=\operatorname{Re}\left(V_{1} I_{12}^{*}\right)+\operatorname{Re}\left(V_{1} I_{13}^{*}\right)
$$

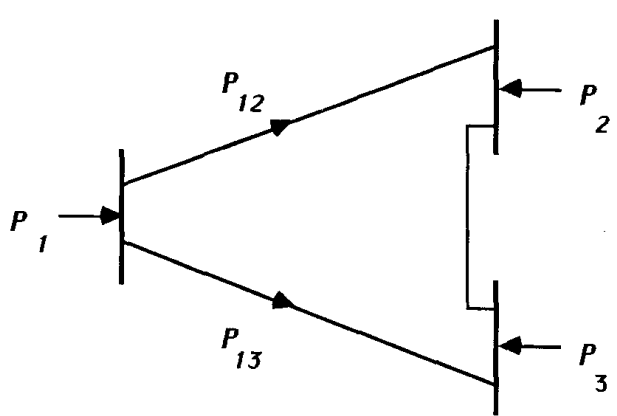

Figure 6. A 3 bus example.

All $V_{i}$ 's, $I_{i}$ 's and $I_{i j}$ 's are variable and their increments can be limited to produce a good feasible directions.

\section{Zoutendijk's Optimization Technique}

This method is a feasible direction technique for optimization of nonlinear programming techniques. The method is based on a successive linearization process that uses an incremental model to produce a feasible improving direction. A complementary line search is then performed along this direction to obtain the optimum for the nonlinear objective without violating nonlinear constraints. This process is continued until the solution converges within a specified accuracy limit. The steps of the method are as follows:

Step 1. Compute the gradient vector $[\nabla f]$ at the existing solution $\left[x_{o}\right]$

Step 2 . Find the feasible improving direction $[d]$ by solving the LP problem, equation (6)-(10).

Step 3. Determine the optimal step $\alpha_{\text {opt }}$ along the direction $[d]$ by minimizing $f\left(x_{o}+\alpha[d]\right)$ subject to feasibility of all constraints (only the nonlinear ones need to be checked). The Golden Search method is used in this optimization together with a feasibility check subroutine. The criterion is to find $\alpha_{\text {opt }}$ so that none of the constraints is violated. Therefore, the values of $\alpha$ that violate a constraint are discarded.

Step 4. $x_{\text {new }}=x_{o}+\alpha_{o p t}[d]$ : if the convergence criterion is not satisfied go to step 1 .

The method of parallel tangents is employed to prevent zigzagging. This method performs an extra line search in the direction constructed by every second solution point. This can be simply demonstrated on figure 8 . The slow convergence apparent on this figure is due to the poor directions from solution point 1 to 2 , from solution point 2 to 3 , etc. The zig-zagging caused by the poor direction can be eliminated by performing a line search in the direction constructed by connecting every second solutions. For example, if a line search is performed in the direction obtained from connecting solution point 1 to 3 (as shown by the dotted line in figure 7), the zig-zagging is eliminated. This is obvious from the figure. The extra line search is very economical and requires very low CPU time compared to the LP solutions.

\section{Feasible Optimal Directions}

A sparse linear programming technique [19] is used to solve the incremental model, equations (6)-(10), which is simplified in this section as follows:

Minimize

$$
c \Delta x
$$

Subject to

$$
A \Delta x=b
$$

$$
\Delta x \varepsilon \Omega
$$




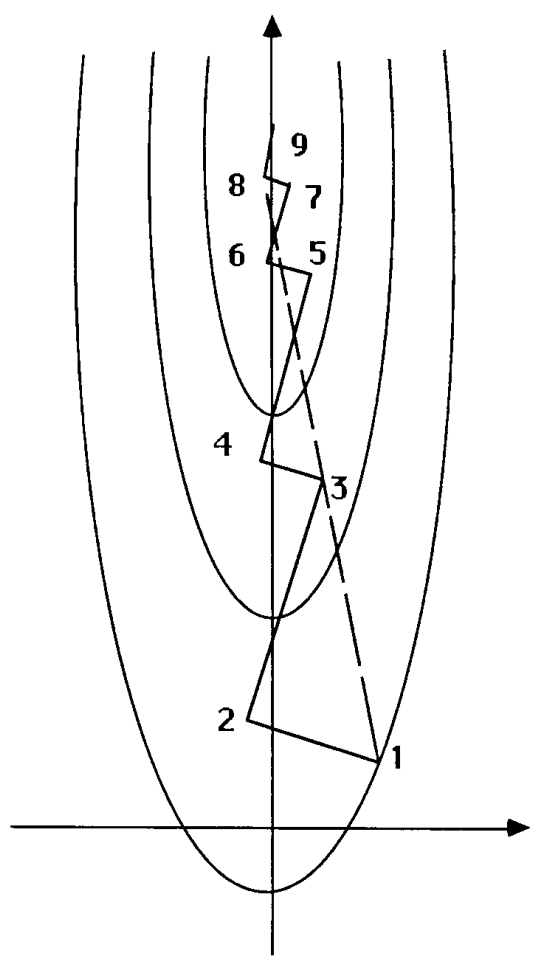

Figure 7. Illustration of the convergence properties of the method

Several important points are considered to reduce the CPU time required in the phase I of this LP which constitutes a major part of the computation time. The phase I of standard LP starts with an all artificial initial solution. This means that one artificial variable per constraint is added to the LP problem:

$$
A \Delta x+s=b
$$

where vector $s$ contains the artificial variable that constitutes the first basic solution. During the phase I process all the variables in $s$ are eliminated to produce a feasible solution for the phase II of LP.

The first point that can be considered to reduce the CPU time required for phase $I$ is the fact that the right hand side vector, $b$ in equation (12), consists of mainly zero elcmients $(90 \%)$ for the OPF. Therefore, an initial solution such as

$$
\Delta x=0
$$

will be feasible for most of the constraints in the problem. Thus, the artificial variables are only required for a few of the constraints in the problem. This reduces the CPU time of phase I to $40-60 \%$ of an all artificial start.

A second point is that the LP basis from each iteration of Zoutendijk's optimization method can be used as the starting basis for the next one. The old basis would be feasible for most of the constraints $(95 \%)$. Artificial variables can be used for the constraints that are not feasible. This reduces the CPU time of the later iterations to $10 \%$ of an all artificial start in the first iteration.

The new binding constraints can be added to the previous basis by simply expanding the basis [24] and adding one artificial variable per new constraints. This procedure requires a very low CPU time.

The points considered above reduce the CPU time requirement of the later iterations in Zoutendijk's optimization technique to a very small fraction of the first iteration. The CPU time of the first iteration is also reduced as compared to a standard all artificial start.

The basis for the Linear Programming problem produced by linear- ization in each step is very sparse. Thus sparse matrix techniques can be used to reduce the storage requirement for the basis matrix. The method of J. K. Reid [19] for handling sparse linear programming basis has been used in this work.

The LP basis has also an embedded network structure. About $45 \%$ of the constraints in this LP problem have a network structure. The above mentioned sparsity techniques have been extended to consider the network structure of the constraints in the problem. The basic idea used in this extension is that the coefficient matrix $A$ corresponding to a problem that has a network structure is actually an incidence matrix. The basis corresponding to this matrix can be triangularized by proper permutations (row column interchanges). Therefore, the operations for the part of matrix with network structure can be simplified with a considerable reduction of computing time.

Other methods, such as dual LP and network flow based techniques, have been examined for solving the incremental model. The main problem with the dual LP technique is that the benefits arising from the embedded network structure are lost. Partitioning and decomposition methods have also been examined for exploiting the embedded network structure of the incremental model. The problem encountered in the partitioning method is that the LP basis from one iteration is not feasible for the next one and causes considerable CPU increases in applying this technique. The decomposition technique on the other hand has slow convergence and can basically be used when the constraints with network structure constitute a major portion of the total set of constraints.

\section{Feasibility Adjustment}

The standard power flow techniques can not consider some system limits, e.g. transmission line limits. Therefore the solution obtained in the first step might not be feasible. These infeasibilities are minimized in the second step.

Consider a line flow limit that is violated:

$$
I>I_{\max }
$$

In order to make this constraint feasible, a feasibility slack variable $S$ can be added as follows:

$$
I-S=I_{\max }
$$

where

$$
S=I-I_{\max }
$$

If the minimization of the variable $S$ is set to be the objective of the LP incremental model, then the objective should be equal to zero for a feasible solution:

$$
S=0
$$

and

$$
I=I_{\max }
$$

Equation (15) is originally nonlinear, therefore it is linearized in the LP incremental model. Consequently, equation (18) might only be feasible in the linearized form. Of course, in such a case the infeasibilitics of the nonlinear constraints are reduced, but not to zero. In the complementary line search the original nonlinear equation is considered and its infeasibility is further reduced. This procedure is repeated until feasibility is obtained. Normally one iteration is required in this step. Since a new itcration requires a low CPU time compared to the first one, it is not a problem to have a few iterations in this step.

\section{Optimization of Objective}

The optimization of objective as shown is figure 5 , is performed in two stages. In the first stage, the optimization is achieved using the piccewise linear approximation of the thermal production curves. This stage does not normally involve a complementary line search due to the fact that the objective is linear. But new linearizations are required until convergence is obtained. This step normally requires $2-3$ iterations. A near optimum solution is obtained in this stage.

In the second stage, the nonlinear objective with quadratic approximation of production costs for the thermal plants is used to obtain further 
improvements. The incremental model is employed to produce a feasible improving direction. A complementary line search is then performed to find the nonlinear optimum along this direction with respect to the nonlinear constraints. This procedure is repeated until convergence within a specified accuracy limit is obtained.

The method applied in this second stage requires 5 to 10 iterations and is very efficient from a near optimum solution such as the one obtained in the first stage optimization.

\section{Optimization Flow Chart}

The optimization flow chart is shown in figure 8. In the first step a power flow solution is obtained by fixing the control variables at the generation buses to some predefined values. The problem is then linearized around this solution. If any of the system limits are violated the feasibility procedure is started. In this procedure the objective is to minimize the infeasibilities of the current solution subject to the equality and binding inequality constraints. Zoutendijk's technique as described earlier (in four steps with the extra line search) is applied. The line search domain in this technique stretches as far as possible with no new constraints being violated. If the infeasibilities are not reduced to zero, the problem is linearized around the new solution and the procedure is repeated until feasibility is obtained. The new binding constraints are added to the active set in each iteration and the LP basis is expanded correspondingly.

Once feasibility is obtained, the problem is linearized and the optimization of generation costs is performed in two stages. In the first stage, the piecewise linear approximation of the cost curve is used producing a simple LP. This stage consists of only one iteration and produces a close optimum solution. The solution from this first stage is used

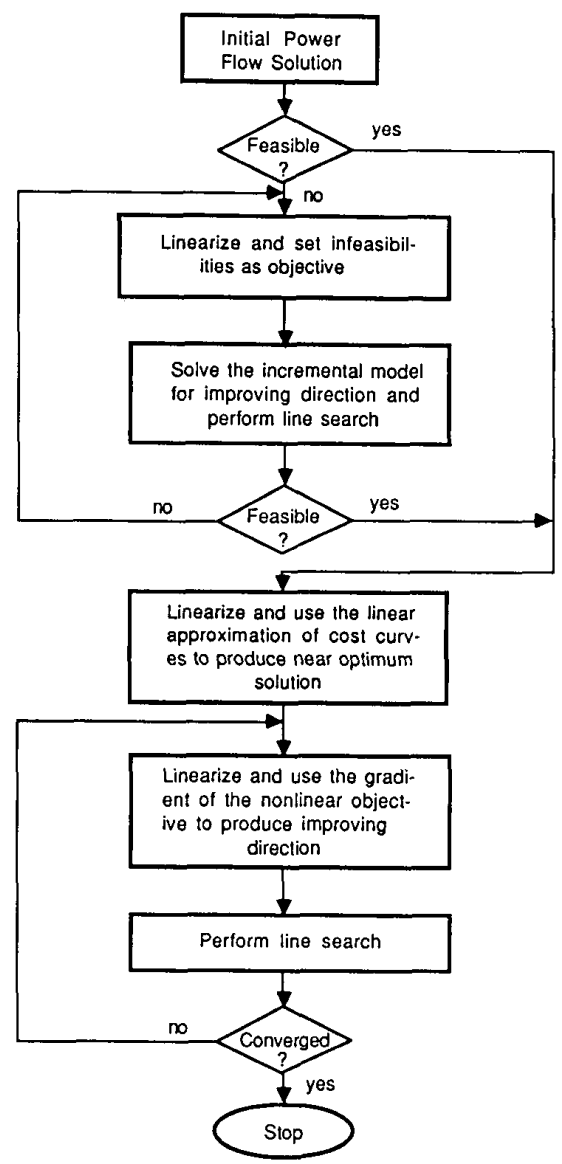

Figure 8. The Optimization Flow Chart. as the starting point for the optimization of the nonlinear objective using Zoutendijk's technique. This second stage optimization consists of several linearizations, direction calculations, and line searches. The procedure is stopped when the desired convergence is obtained. The convergence criterion is defined on the improvements from one iteration to the next.

\section{TEST RESULTS}

A computer program has been developed and tested. The procedure proved to be very efficient since it exploits the special structure of the problem. The IEEE 39 bus test system, shown in figure 9 , is used in this section for presentation of the results. Two river systems consisting of seven power plants have been added to the system. The transmission network connections of the first river system are at nodes 10,11 and 13 and the second river system connections are at nodes $7,9,12$ and $17 .^{\circ}$ The maximum plant productions in the hydraulic system varies from 1.2 to 4.2 p.u. in a 100 MVA power base, while the maximum thermal plant productions which vary from 3.5 to 7.5 p.u. The system consists of 46 branches. Table 1 summarizes some interesting results obtained on a VAX 8600 . Phase I and II are compared in the first and following iterations. The standard all artificial phase I start is compared with the partial artificial start used in this method. The method starts from zero increments

$$
\Delta x=0
$$

Table 1. Some test results on the 39-bus system.

\begin{tabular}{|l|c|c|c|}
\hline \multicolumn{1}{|c|}{ Description } & $\begin{array}{c}\text { No. of pivot } \\
\text { operations } \\
\text { in LP }\end{array}$ & $\begin{array}{c}\text { CPU } \\
\text { time } \\
\text { seconds }\end{array}$ & $\begin{array}{c}\text { LP size } \\
\text { No. of basic } \\
\text { variables }\end{array}$ \\
\hline $\begin{array}{l}\text { Phase I, iteration 1 } \\
\text { (all artificial start) }\end{array}$ & $700-1000$ & $2-6$ & $420-430$ \\
\hline $\begin{array}{l}\text { Phase I, iteration 1 } \\
\text { (partial artificial start) }\end{array}$ & $300-600$ & $0.5-4$ & $420-430$ \\
\hline Phase II, iteration 1 & $10-20$ & - & $420-430$ \\
\hline Phase I, other iterations & $20-200$ & $<1$ & $420-430$ \\
\hline Phase II, other iterations & $10-60$ & - & $420-430$ \\
\hline Total Zoutendijk technique & $10-20$ & $5-15$ & - \\
\hline
\end{tabular}

An all artificial Phase I start in iteration 1 consumes a major part of the computation time required to solve the problem. The CPU time required for the first iteration is equivalent to the CPU time required by the following 5 to 10 iterations. This is very important in such an iterative method.

The convergence of the proposed technique is demonstrated in Table 2. The costs are normalized with respect to the initial solution cost. The costs increase in the feasibility stage and decrease later during the optimization process. The number of binding inequality constraints also increases during the procedure.

The total CPU time required for solving the 39 -bus system is 5 to 15 seconds depending on the operational limits considered. The total CPU time required for a 118 bus system, the largest system considered in this study, was 30-45 seconds. This system included 3 river systems 


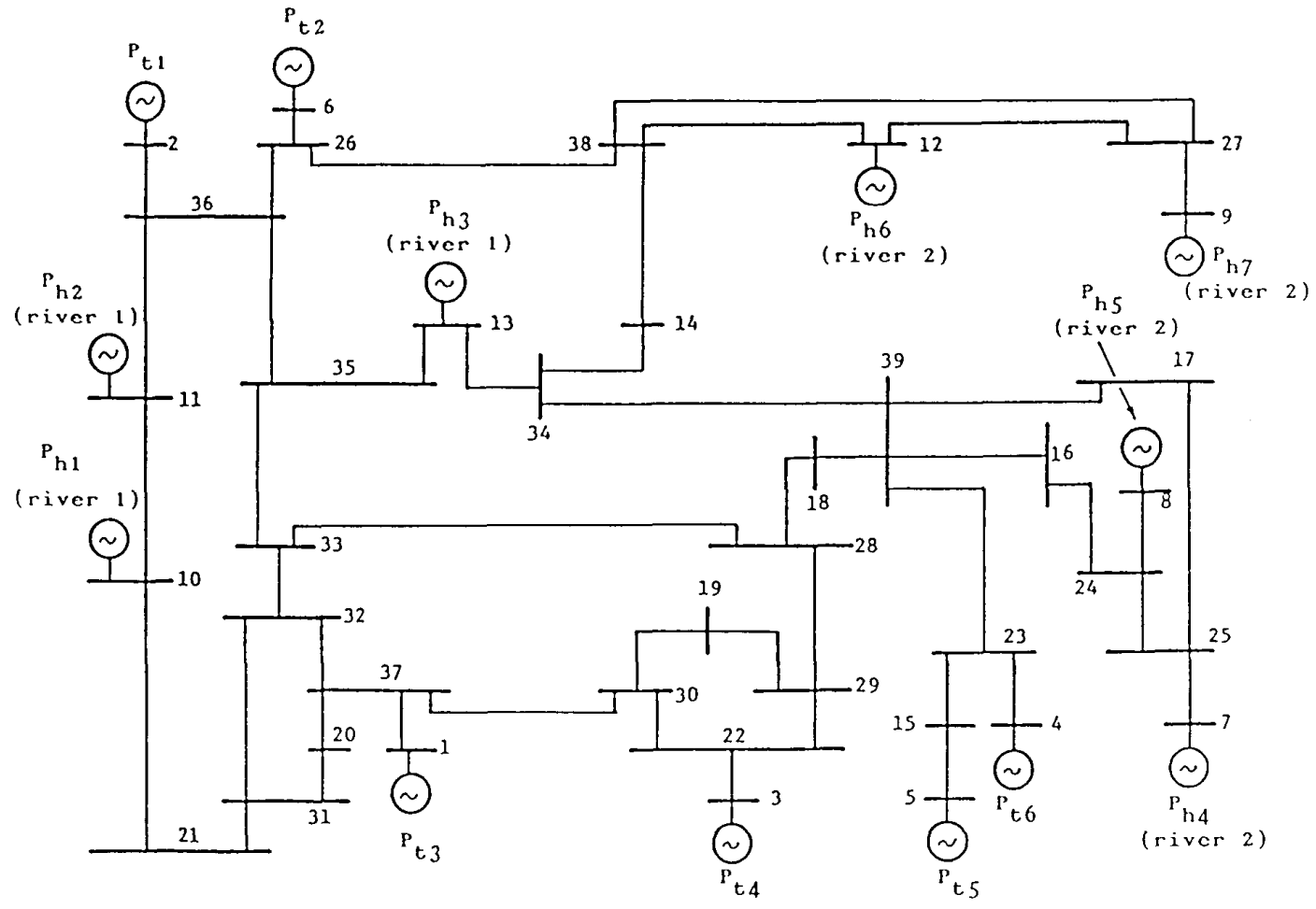

Figure 9. The 39-bus system.

Table 2. Some test results on the convergence of 39-bus system.

\begin{tabular}{|r|c|c|c|c|}
\hline Stage & LP size & $\begin{array}{c}\text { No. of } \\
\text { binding } \\
\text { inequalities }\end{array}$ & $\begin{array}{c}\text { Total system } \\
\text { cost (norm.) }\end{array}$ & $\begin{array}{c}\text { CPU time } \\
\text { (seconds) }\end{array}$ \\
\hline Power flow & - & - & 1.0 & - \\
\hline Feasibility & 422 & 2 & 1.1 & 3.0 \\
\hline Approx. opt. & 422 & 2 & 0.89 & 0.6 \\
\hline Nonlinear opt. & & 4 & 0.85 & 0.5 \\
iter. 1 & 422 & 2 & 0.83 & 0.8 \\
iter. 2 & 422 & 3 & 0.81 & 0.5 \\
iter. 3 & 423 & 3 & 0.80 & 0.8 \\
iter. 4 & 424 & 4 & 4 & 0.8 \\
iter. 5 & 424 & 4 & & 0.8 \\
\hline
\end{tabular}

consisting of 25 hydraulic stations.

Comparison of this method with previous ones reported in the literature highlights its capability of integrating the OPF into a system with strong hydraulic content.

\section{CONCLUSIONS}

A new formulation of Optimal Power Flow has been presented in this work. This formulation is branch oriented as opposed to the conventional nodal approach. The model for this formulation is very sparse and has an embedded network structure. Zoutendijk's feasible directions technique was employed to solve the nonlinear programming problem exploiting the special structure of the problem. The hydraulic units were modeled to reflect their importance in a system with considerable share of hydraulic gencration.

The Zoutendijk optimization technique was further accelerated by taking into account the followings:

1) The piecewise linear approximation considered in the first stage produces a near optimum starting point for the nonlinear optimization. Therefore it eliminates an excessive number of initial iterations in the nonlinear solution.

2) The method of parallel tangents eliminates slow convergence that might result from zig-zagging.

3) The LP solution for the incremental model in each iteration differs from the next one by only few equations. Therefore, the basic solution obtained in one iteration is used in the next one to accelerate the optimization technique.

4) An initial zero incremental solution is used in the phase I of iteration one to reduce the computation time. This transforms an all artificial start to a partial one.

5) The embedded network structure of the constraints is used to speed up the basis manipulations.

6) The new binding constraints which are not active at the initial solution are added later, when they bind, in a simple manner with low contributions to CPU time.

The specific characteristics of this work are:

1) The method is particularly efficient for constrained OPF problems, since it can start with an active set of constraints and add the new 
binding constraints in a simple manner so that the increase of CPU time is very low.

2) The program can start from an infeasible initial solution.

3) The hydraulic system is taken into account for a power system with considerable amount of hydraulic generation.

4) All components of the power system and their limits can be considered in a very simple formulation.

5) The expanded branch oriented formulation used in this work makes it possible to produce an exact incremental model and consequently very good optimum feasible directions. This renders the solution technique very efficient.

\section{ACKNOWLEDGEMENTS}

The authors acknowledge the financial support for this work by a grant from the Natural Science and Engineering Research Council of Canada. Support and encouragement by Drs. M. El-Kady and D. Frances from the Resource Utilization Department of Ontario Hydro are highly appreciated.

\section{REFERENCES}

[1] J. L. Carpentier, "Differential Injections Method, a General Method for Secure and Optimal Load Flows", Proc. of 8th PICA Conf., pp. 225-262, Minneapolis, MN, 1973.

[2] H. W. Dommel and W. F. Tinney, "Optimal Power Flow Solutions", IEEE Trans. on Power Apparatus and Systems, Vol. PAS87, pp. 1866-1876, October 1968.

[3] J. Peschon, et al, "Optimal Solutions Involving System Security", Proc. 7th PICA conf., pp. 210-218 Boston, Mass., 1971.

[4] Y. Backlund and D. Sjelvgren, " An Extended Generalized Gradient Methodology with Second Order Information for Optimal Power Flows", Proc. of IEE conference on Power System Monitoring an Control, Durham, England, July 1986.

[5] A. M. Sasson and H. M. Merrill, "Some Applications of Optimization Techniques to Power System Problems", Proc. of IEE, Vol. 62 pp. 959-972, July 1974.

[6] H. H. Happ, "Optimal Power Dispatch - A Comprehensive Survey", IEEE Trans. on Power Apparatus and Systems, Vol. PAS-96, No. 3, pp. 841854 , May/June 1977.

[7] B. F. Wollenberg and W. O. Stadlin, "A Real Time Optimizer for Security Dispatch", IEEE Trans. on Power Apparatus and Systems, Vol. PAS-93, pp. 1640-1649, Sept/Oct 1974.

[8] B. Stott and E. Hobson, "Power System Security Control Calculation Using Linear Programming, Part I and II", IEEE Trans. on Power Apparatus and Systems. Vol. PAS-97, pp. 1713-1731, Sep/Oct 1978.

[9] E. Hobson, "Network Constrained Reactive Power Control Using Linear Programming", IEEE Trans. on Power Apparatus and System, Vol. PAS-99, No.3, pp. 868-877, May/June 1980.

[10] B. Stott and J. L. Marinho, "Linear Programming for Power System Network Security Applications", IEEE Trans. on Power Apparatus and System, Vol PAS-98, No. 3, pp. 837-848, May/June 1979.

[11] K. R. C. Mamandur and R. D. Chenoweth, "Optimal Control of Reactive Power Flow for Improvement in Voltage Profiles and Real Power Loss Minimization", IEEE Trans. on Power Apparatus and System, Vol. PAS-100, No.7, pp. 3185-3194, July 1981.

[12] N. Nabona and M. J. Freris, "Optimization of Economic Dispatch through Quadratic and Linear Programming", Proc. IEE, Vol 120 , pp. 574-579, May 1973.

[13] H. Nicholson and M. J. Streling, "Optimum Dispatch of Active and Reactive Generation by Quadratic Programming", IEEE Trans. on Power Apparatus and Systems, Vol. PAS-92, No. 2, pp. 644-654,
Mar/Apr 1973.

[14] W. O. Stadlin and D. L. Fetcher, "Voltage Versus Reactive Current Model for Dispatch and Control", IEEE Trans. on Power Apparatus and System, Vol. PAS-101, No. 10, pp. 3751-3760, Oct 1982.

[15] J. S. Horton and L. L. Grigsby, "Voltage Optimization Using Combined Linear Programming \& Gradient Techniques", IEEE Trans. on Power Apparatus and Systems, Vol. PAS-103, No.7, July 1984.

[16] R. C. Burchett, H. Happ and D. R. Vierath, "Quadratically convergent Optimal Power Flow", IEEE Trans. on Power Apparatus ans System, Vol. PAS-103, pp. 3267-3275, Nov. 1984.

[17] M. S. Bazaraa, C. M. Shetty, "Nonlinear Programming", John Wiley \& Sons, New York, 1979.

[18] L. S. Lasdon, "Optimization Theory for Large Systems", Macmillan Co., 1970.

[19] J. K. Reid, "Fortran Subroutines for Handling Sparse Linear Programming Basis", Report A.E.R.E.-8269, Harwell, England, January 1976.

[20] J. L. Kennigton and R. V. Helgason, "Algorithm for Network Programming", John Wilcy \& Sons, N.Y. 1981.

[21] G. Zoutendijk, "Methods of Feasible Directions", Elsevier Publishing Co., Amsterdam, 1960

[22] D. Sjelvgren, et al, "Optimal Seasonal Operation Planning in a Large Scale Hydrothermal Power System", IEEE Trans. on Power Apparatus and Systems, Vol. PAS-101, No. 11, pp. 3644-3651, Nov. 1983.

[23] H. Habibollahzadeh and J. Bubenko, "Application of Decomposition Technique to Short-Term Operation Planning of Hydrothermal Power System, Vol. PWRS-1, No. 1, pp. 41-47, Feb. 1986.

[24] A. H. Land and S. Powell, "Fortran Codes for Mathematical Programming: Lincar, Quadratic and Discrete", John Wiley \& Sons, London, 1973.

[25] M.E. El-Hawary and D.H. Tsang, "The Hydrothermal Optimal Load Flow: A Practical Formulation and Solution Techniques using Newton's Approach", IEEE Trans. on Power Systems, Vol. PWRS-1, No. 3, Aug. 1986, pp. 157-167.

[26] G.A. Maria and J.A. Findlay, "A Newton Optimal Power Flow Program for Ontario Hydro, IEEE Trans. on Power Systems, Vol PWRS-2, No. 3, August 1987.

Hooshang Habibollahzadeh (member of IEEE) was bom in Iran on September 15, 1951. He received his B.S.E.E and M.S.E.E from the University of Washington, Seattle, USA, in 1973 and 1974 respectively. He was a lecturer at the Iran University of Science and Technology, Tehran, Iran, 1975-1981. In 1981 he joined the Encrgy Systems Laboratory at the Royal Institute of Technology, Stockholm, Sweden, as a research assistant where he received his Ph.D. in 1984. He was a lecturer and researcher at this Institute 1984-1986. He was a research associate at the University of Toronto, Toronto, Canada, 1986-1987. Recently, he has joincd the Ontario Hydro Power System Operation Department.

Guang-Xiang Luo was born in Canton, China on August 22, 1946. $\mathrm{He}$ received B.A.Sc. degree from Wuhan Institute of Hydraulic and Electric Engineering, China in 1970, the M.A.Sc. and Ph.D. degrees in electrical engineering from University of Toronto, Canada in 1983 and 1988 , respectively. From 1970 to 1978 , he was employed by Fong-Man Power station, Northeast China. He worked at the station control center as a graduate enginecr.

Adam Semlyen (fellow of IEEE) was born and educated in Rumania where he obtained a Dipl. Ing. degree and his Ph.D. He started his career with an electric power utility and held an academic position at the Polytechnic Institute of Timisoara, Rumania. In 1969 he joined the University of Toronto where he is a professor in the Department of Electrical Engineering. His research interests include the Steady State and Dynamic Analysis of Power Systems, Electromagnetic Transients, and Power System Optimization. 